\title{
Posthaemorrhagic ventricular dilatation in the premature infant: natural history and predictors of outcome
}

\author{
B P Murphy, T E Inder, V Rooks, G A Taylor, N J Anderson, N Mogridge, L J Horwood, \\ J J Volpe
}

Arch Dis Child Fetal Neonatal Ed 2002;87:F37-F41

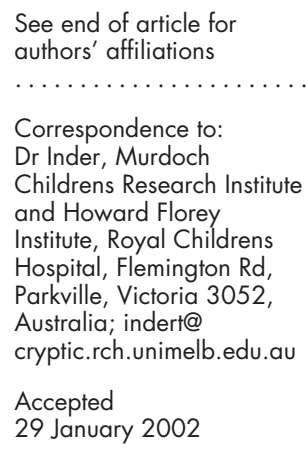

\begin{abstract}
Objective: To investigate the natural history and predictors of outcome of posthaemorrhagic ventriculomegaly in the very low birthweight (VLBW) infant.

Methods: All VLBW infants admitted between September 1994 and September 1997 to the neonatal intensive care units of Brigham and Women's Hospital (Boston), Children's Hospital (Boston), and Christchurch Women's Hospital (New Zealand) with germinal matrix intraventricular haemorrhage (IVH) were identified. All charts and ultrasound scans were reviewed to define the natural history and perinatal and/or postnatal factors of value in prediction of the course of posthaemorrhagic ventriculomegaly. Progressive ventricular dilatation (PVD) was defined from the results of serial cranial ultrasound scans.

Results: A total of 248 VLBW infants had evidence of IVH (22\% of all VLBW infants, mean (SD) gestational age 26.8 (2.6) weeks). A quarter of the infants exhibited PVD. Spontaneous arrest of PVD occurred without treatment in 38\% of infants with PVD. Of the remaining 62\% with persistent PVD, $48 \%$ received non-surgical treatment only (pharmacological and/or drainage of cerebrospinal fluid by serial lumbar punctures), 34\% received surgical treatment with insertion of a ventriculoperitoneal reservoir and/or shunt, and $18 \%$ died. The development of PVD after IVH and adverse short term outcome, such as the requirement for surgery, were predicted most strongly by the severity of IVH.

Conclusions: These data reflect the natural history of PVD in the 1990s and show that, despite a slight reduction in its overall incidence, there appears to be a more aggressive course, with appreciable mortality and morbidity in the extremely premature infant. The major predictor of adverse short term outcome, defined as death or need for surgical intervention, was the severity of IVH. These findings may be valuable for the management of very small premature infants.
\end{abstract}

u mprovements in perinatal and neonatal care have contributed to a reduction in the overall incidence of intraventricular haemorrhage (IVH) in very low birthweight (VLBW, birth weight $<1500 \mathrm{~g}$ ) premature infants from $40-50 \%$ in the later 1970 s and 1980 s to $20-25 \%$ in the 1990 s. $^{12}$ However, the 1990 s have also seen increased survival of the extremely premature infant (birth weight $<750 \mathrm{~g}$ ), who is at greatest risk of severe IVH and subsequent progressive posthaemorrhagic ventricular dilatation (PVD). ${ }^{3}$ The morbidity of PVD is appreciable. Up to $90 \%$ of infants have subsequent neuromotor disability, $76 \%$ exhibit pronounced disability, and 56\% have multiple impairments. ${ }^{4}$ Studies of the natural history of PVD in the late 1980s suggested that about one third of infants with IVH develop PVD. ${ }^{5}$ Of VLBW infants with PVD, 85\% subsequently had arrest of progression, spontaneous or induced by non-surgical intervention, and 15\% required ventriculoperitoneal (VP) shunts for control of raised intracranial pressure. ${ }^{5}$ With an increased proportion of extremely preterm infants now surviving, the aim of this study was to define the incidence and natural history of PVD in VLBW infants in the 1990s and to define predictive factors for adverse outcome.

\section{METHODS}

\section{Subjects}

All VLBW infants with IVH admitted between September 1994 and September 1997 to the newborn intensive care units at Brigham and Women's Hospital and Children's Hospital in Boston and Christchurch Women's Hospital in New Zealand were identified. Diagnostic coding of IVH on neonatal discharge summaries was cross referenced with radiology records of all infants in each unit with a diagnosis of IVH on any cranial ultrasound report during the study interval. Cases identified were cross referenced against all infants with a birth weight of $1500 \mathrm{~g}$ or less admitted to each intensive care unit to ensure the validity of the cohort sample. Infants with chromosomal abnormality, congenital heart disease (patent ductus arteriosus excepted), and identified congenital abnormality of the central nervous system were excluded from the study. The mean (SD) gestational age of our cohort was 26.8 (2.6) weeks with a mean (SD) birth weight of 950 (306) g. The institutional research review boards approved this study in all the participating centres.

\section{Data collection}

Case records were reviewed independently by two neonatologists (BPM and TEI). Basic information, gestational age, birth weight, and perinatal factors were recorded. A CRIB score, a physiological measure of stability based on blood pressure, oxygen requirement, gestational age, birth weight, and presence of congenital abnormality in the first 12 hours of life, was calculated for each infant. ${ }^{6}$ Postnatal variables including measures of physiological derangement in the first 48 hours $^{7}$ and outcome were collected.

\section{Cranial ultrasound scans}

In these units, cranial ultrasound scans were carried out routinely on all infants of birth weight $>1500 \mathrm{~g}$. The scans were

Abbreviations: IVH, intraventricular haemorrhage; VLBW, very low birthweight; PVD, progressive ventricular dilatation; VP, ventriculoperitoneal 
performed during the first five days of life and then in the second and sixth weeks of life. If any abnormality was identified, they were carried out on at least a weekly basis. For this study, in each site, every scan on each infant was re-evaluated by an experienced radiologist (VR, GAT, and NJA) blinded to the infant's perinatal details, to confirm the reported ultrasonographic findings and to quantify the ventricular dilatation.

Cranial ultrasound scans were carried out via the anterior fontanelle in standard coronal and parasagittal views. IVH was classified as grades $\mathrm{l}$ to 4 according to Volpe's classification. ${ }^{5}$ Grade 1 represents germinal matrix haemorrhage only with no obvious IVH. Grade 2 represents IVH occupying $10-50 \%$ of ventricular area on parasagittal view. Grade 3 is IVH with haemorrhage occupying $>50 \%$ of ventricular area on parasagittal view. Grade 4 represents severe IVH with associated periventricular echodensity. Ventricular enlargement was qualitatively classified as mild, moderate, or severe for the lateral, third, and fourth ventricles on each ultrasound scan. Quantitative measurements of ventricular enlargement were recorded on each successive cranial ultrasound scan on each infant in the following standardised manner. The width of the anterior horn of the lateral ventricles was measured in a coronal view, with the plane of the scan at the level of the foramina of Monro (just anterior to the choroid plexus in the third ventricle). The width was measured on each side as the distance between the medial wall and the floor of the lateral ventricle at the widest point. ${ }^{7}$ The reference range for this dimension in healthy preterm infants of 23-33 weeks gestation has been defined as $0-2.9 \mathrm{~mm}^{7}$ Periventricular white matter injury was classified by a modified grading system as either localised (frontal, parietal, or parieto-occipital) or extensive (frontal to parieto-occipital) echodensity or echolucency..$^{58}$

The population with posthaemorrhagic ventricular dilatation was defined as those infants with IVH whose ventricles were dilated at any stage on cranial ultrasound scan subsequent to the haemorrhage identified on the initial cranial ultrasound scan. Mild ventricular dilatation was defined qualitatively by fullness in the lateral ventricles with rounding of the anterior frontal horns. The median quantitative anterior horn width for mild ventricular dilatation was 6 $\mathrm{mm}$ (range 4-10). Moderate ventricular dilatation was defined qualitatively as dilatation in the lateral ventricular system with ventricular effacement and loss or reduction of the subarachnoid space. The median quantitative anterior horn width for moderate ventricular dilatation was $14 \mathrm{~mm}$ (range 8-18). Marked ventricular dilatation was defined as dilatation including the lateral ventricular, third, and fourth ventricles with more pronounced ventricular effacement and loss of the subarachnoid space. The median quantitative anterior horn width for marked ventricular dilatation was $22 \mathrm{~mm}$ (range 12-36). PVD was defined by either a rapid severe increase in lateral ventricle size on cranial ultrasound within seven days of IVH or the continued progression of ventricular dilatation identified on cranial ultrasound for more than 14 days after IVH characterised by a change in qualitative classification (from mild to moderate or severe ventricular dilatation) or quantitatively by an increase in anterior horn width of at least $6 \mathrm{~mm}$. The median maximal anterior horn width for infants with PVD was $15 \mathrm{~mm}$ (range 8-36).

\section{Treatments}

Non-surgical treatment was defined as pharmacological interventions (acetazolamide and/or furosemide) and serial lumbar punctures to remove cerebrospinal fluid for the treatment of PVD (this did not include diagnostic taps performed for diagnosis of sepsis). Surgical treatments for PVD were either (a) ventriculostomy with placement of a ventricular catheter and subcutaneous reservoir for continuous/ intermittent ventricular drainage, or $(b)$ VP shunt. Timing of all treatments, medical or surgical, and all complications of treatments was recorded.

\section{Statistical analysis}

Statistical analyses were performed with SyStat and SPSS for Windows (SPSS Inc, Chicago, Illinois, USA). The infants with IVH surviving beyond 14 days of life were classified into three groups on the basis of the severity of ventricular dilatation: no ventricular dilatation, ventricular dilatation without progression, and PVD. For comparison of the measures of perinatal status between the three groups, tests of significance were based on the $\chi^{2}$ test of independence for dichotomous measures and one way analysis of variance for continuous measures. To examine the factors that predicted outcome severity, the data were analysed using an ordinal logistic regression model in which the three level outcome severity indexes were regressed on potential predictors in two stages, first regressing the outcome on predictors other than grade of IVH and then including grade of IVH. The characteristics of the 19 infants who had surgical intervention for IVH were compared with those of the remaining 202 infants, using the $\chi^{2}$ test of independence for dichotomous measures and one way analysis of variance for continuous measures. As discussed below, only two variables were significantly related to the likelihood of surgery-that is, grade of IVH $(\mathrm{p}<0.0001)$ and inotropic support $(\mathrm{p}<0.005)$-and these variables were entered into a logistic regression model to predict the risk of surgery.

\section{RESULTS \\ IVH}

A total of 248 VLBW infants had evidence of IVH. The overall incidence of all grades of IVH among VLBW admissions to the three units in aggregate was $22 \%$. Severe IVH (grade $3 /$ IV) accounted for $32 \%$ of all IVH. The mean (SD) gestational age of the infants with severe IVH was 26.0 (1.9) weeks. Of note, $82 \%$ of cases with grade 3 IVH were bilateral.

\section{Mortality}

Fifty four infants (22\%) died, with half of the deaths occurring in the first 14 days of life. Two thirds of all deaths in the first 14 days $(60 \%$ of all deaths) occurred in infants with severe IVH (grade 3/IV) (table 1).

\section{Ventricular dilatation}

A total of 221 VLBW infants with IVH survived beyond 14 days and thus were eligible for analysis of the presence of ventricular dilatation. Of these 221 infants, 112 (51\%) showed no ventricular dilatation at any stage (fig 1A). The remaining 109 infants $(49 \%)$ showed ventricular dilatation on at least one cranial ultrasound scan after the IVH. Fifty three infants $(24 \%)$ showed no significant change in ventriculomegaly qualitatively or quantitatively. The median anterior horn width was $6 \mathrm{~mm}$ (range 4-9). These infants were classified as having non-progressive ventricular dilatation (fig 1 ). Fifty six infants $(25 \%)$ developed PVD on serial cranial ultrasound scans after IVH (fig lA). Of particular note, 45 of these 56 infants $(80 \%)$ had severe (grade $3 / 4)$ IVH.

\section{Treatment for PVD}

Of the 56 infants with PVD, 21 infants (38\%) did not receive any treatment and had spontaneous arrest and/or resolution of their PVD (fig lB). The remaining 35 infants (62\% of PVD) had persistent PVD. Six of these infants died before receiving treatment for PVD, with death resulting principally from various systemic complications of prematurity. Thus, 29 infants were treated for persistent PVD. In all but two, management was non-surgical-that is, pharmacological agents (acetazolamide and/or diuretics) or serial lumbar punctures or both. Thus 20 infants received combined pharmacological treatment and serial lumbar punctures, six infants had serial lumbar punctures alone with no accompanying pharmacological treatment, one infant had pharmacological treatment with no 
Table 1 Short term outcome of germinal matrix intraventricular haemorrhage (IVH) as a function of severity of haemorrhage in infants weighing $<1500 \mathrm{~g}$ at birth

\begin{tabular}{|c|c|c|c|c|c|c|c|c|}
\hline \multirow[b]{2}{*}{ Grade of IVH } & \multicolumn{2}{|c|}{ Deaths in first 14 days } & \multicolumn{2}{|c|}{$\begin{array}{l}\text { PVD in survivors }>14 \text { days } \\
\text { old }\end{array}$} & \multicolumn{2}{|c|}{ Surgery + late death } & \multirow[b]{2}{*}{ Mortality } & \multirow[b]{2}{*}{$\begin{array}{l}\text { Surgery in } \\
\text { survivors }\end{array}$} \\
\hline & $\begin{array}{l}<750 \mathrm{~g} \\
(\mathrm{n}=75)\end{array}$ & $\begin{array}{l}751-1500 \mathrm{~g} \\
(n=173)\end{array}$ & $\begin{array}{l}<750 \mathrm{~g} \\
(\mathrm{n}=56)\end{array}$ & $\begin{array}{l}751-1500 \mathrm{~g} \\
(\mathrm{n}=165)\end{array}$ & $<750 \mathrm{~g}(\mathrm{n}=56)$ & $\begin{array}{l}751-1500 \mathrm{~g} \\
(\mathrm{n}=165)\end{array}$ & & \\
\hline I $(n=104)$ & $3 / 24(12)$ & $0 / 80(0)$ & $1 / 21(5)$ & $3 / 80(4)$ & $0+4 / 21$ (19) & $0+3 / 80(0)$ & $10 / 104(10)$ & $0 / 94(0)$ \\
\hline II $(n=65)$ & $5 / 21(24)$ & $1 / 44(2)$ & $1 / 16(6)$ & $6 / 43(14)$ & $0+5 / 16(31)$ & $1+2 / 43(7)$ & $13 / 65(20)$ & $1 / 52(2)$ \\
\hline III $(n=45)$ & $6 / 19$ (32) & $2 / 26(8)$ & $10 / 13$ (77) & $18 / 24(75)$ & $3+2 / 13(38)$ & $7+4 / 24(42)$ & $14 / 45$ (31) & $10 / 31$ (32) \\
\hline IV $(n=34)$ & $5 / 11(45)$ & $5 / 23(22)$ & $5 / 6(83)$ & $12 / 18(66)$ & $1+3 / 6(66)$ & $7+4 / 18(61)$ & $17 / 34(50)$ & $8 / 17$ (47) \\
\hline
\end{tabular}

serial lumbar punctures, and only two infants had VP shunt insertion with neither prior pharmacological treatment nor serial lumbar punctures. Pharmacological treatment for PVD began on median day of life 12 (range 6-22), whereas serial lumbar punctures started on median day of life 19 (range 11-36) with a median number of 11 serial lumbar punctures (range 3-33) undertaken. Of the 27 infants with persistent PVD treated pharmacologically or with serial lumbar punctures or both, 17 (63\%) ultimately required a VP shunt for continued progression, eight experienced arrest of progression, and two died from systemic complications of prematurity (fig 1B). Overall, 29 infants (52\% of PVD) had spontaneous or non-surgical treatment induced arrest, and 19 infants (34\% of PVD) required surgical intervention with a ventricular reservoir or VP shunt (fig $1 \mathrm{~B}$ ).

Surgical intervention was carried out on median day of life 35 (range 5-147) in a total of 19 infants (34\% of PVD). Twelve infants had a ventricular reservoir inserted as the initial procedure, and seven had VP shunt insertion as their first surgical procedure. One infant died soon after ventricular reservoir insertion, and 10 of the surviving 11 infants ultimately required a VP shunt. Consistent with their principal role as a temporary measure, reservoirs were inserted earlier-that is, on median day of life 24 -than the initial placement of the VP shunt on median day of life 50 .

Thus, of the 109 infants with ventricular dilatation, 82 (75\%) either did not exhibit progression (49\%) or experienced arrest of progressive dilatation spontaneously (19\%) or after non-surgical treatment (7\%). The remaining 27 (25\%) either eventually required a VP shunt $(17 \%)$ or died $(8 \%)$. The adverse outcome of death or requirement for a VP shunt therefore occurred in $12 \%$ of the starting population of 221 infants with IVH who survived more than 14 days.

\section{Predictors of occurrence and outcome of PVD}

Of the infants with IVH who survived more than 14 days, the occurrence of PVD and the requirement for surgical ventricular drainage were most strongly predicted by severity of IVH (table 1). PVD occurred in $4 \%$ of infants with grade $1 \mathrm{IVH}, 12 \%$ with grade $2 \mathrm{IVH}, 74 \%$ with grade $3 \mathrm{IVH}$ and $71 \%$ with grade $4 \mathrm{IVH}$. As noted earlier, $82 \%$ of grade 3 haemorrhages were bilateral.

Compared with infants who did not develop PVD, those who did were significantly smaller, more immature, sicker in the first 12 hours of life as manifested by a higher CRIB score, and had significantly greater hypercarbia, and a greater number of infants who developed PVD required inotropic support for hypotension (table 2). However, the strongest predictor of the occurrence of PVD was severity of IVH, in particular grade 3/4. There was no significant difference between the three groups with respect to either antenatal corticosteroid treatment or hypertension during the first 48 hours of life.

Ordinal logistic regression analysis was carried in two stages to further examine the predictors of PVD, first regressing the outcome on predictors other than grade of IVH and
A

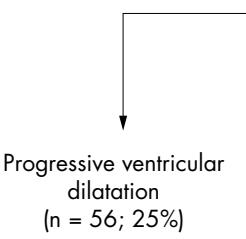

B
$\mathrm{IVH}$ and survival $>14$ days $(\mathrm{n}=221)$

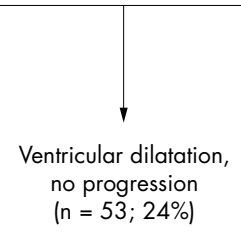

( $\mathrm{n}=53 ; 24 \%$ )

Figure 1 Natural history of very low birthweight infants with posthaemorrhagic ventricular dilatation (PVD). IVH, Intraventricular haemorrhage.

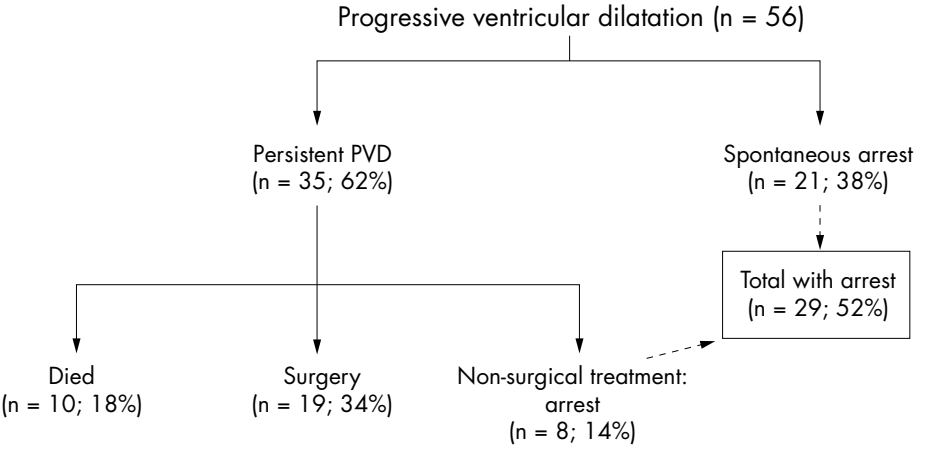


Table 2 Comparison of infants surviving to day 14 and classified by degree of ventricular dilatation on measures of perinatal status

\begin{tabular}{|c|c|c|c|c|}
\hline Measure & $\begin{array}{l}\text { No ventricular } \\
\text { dilatation } \\
(n=112)\end{array}$ & $\begin{array}{l}\text { Non-progressive } \\
\text { ventricular } \\
\text { dilatation } \\
(n=53)\end{array}$ & $\begin{array}{l}\text { Progressive } \\
\text { ventricular } \\
\text { dilatation } \\
(n=56)\end{array}$ & $\mathrm{p}$ Value \\
\hline$\%$ Male & 54.1 & 68.5 & 55.4 & $>0.15$ \\
\hline Gestation (weeks) & $27.9(2.7)$ & $26.2(2.2)$ & $26.2(2.0)$ & $<0.0001$ \\
\hline Birth weight $(\mathrm{g})$ & $1053(285)$ & 965 (297) & $938(273)$ & $<0.05$ \\
\hline CRIB score & $4.6(4.1)$ & $6.0(4.2)$ & $7.1(4.1)$ & $<0.001$ \\
\hline$\%$ Maximum IVH grade 3 or 4 & 0.0 & $29.6^{17.4}$ & 80.4 & $<0.0001$ \\
\hline Maximum $\mathrm{PCO}_{2}{ }^{*}$ & $53.6(14.0)$ & $62.2(16.3)$ & $60.1(15.6)$ & $<0.001$ \\
\hline$\%$ Hypertension & 51.4 & 46.3 & 50.0 & $>0.8$ \\
\hline$\%$ Inotropic support & 45.9 & 63.0 & 75.0 & $<0.001$ \\
\hline$\%$ Antenatal steroids & 81.1 & 77.8 & 69.6 & $>0.20$ \\
\hline \multicolumn{5}{|c|}{$\begin{array}{l}\text { Where applicable, values are mean (SD). The test of significance was based on the } \chi^{2} \text { test of independence } \\
\text { for dichotomous measures and one way analysis of variance for continuous measures. } \\
{ }^{*} \mathrm{PCO}_{2} \text { values for nine children who were not ventilated were set to an arbitrary value of } 40 \text {. Excluding these } \\
\text { nine children does not materially alter the strength of this association. } \\
\mathrm{IVH} \text {, Intraventricular haemorrhage. }\end{array}$} \\
\hline
\end{tabular}

Table 3 Summary of ordinal regression coefficients for significant predictors of progressive ventricular dilatation

\begin{tabular}{lllll}
\hline Measure & $\mathrm{B}(\mathrm{se})$ & $\mathrm{p}$ Value & Odds ratio & $95 \% \mathrm{Cl}$ \\
\hline Gestation & $-16(0.07)$ & $<0.05$ & 0.85 & 0.75 to 0.97 \\
Grade of IVH & $1.66(0.19)$ & $<0.0001$ & 5.27 & 3.62 to 7.63
\end{tabular}

$\mathrm{Cl}$, Confidence interval; IVH, intraventricular haemorrhage.

Table 4 Comparison of infants receiving and not receiving surgical intervention for intraventricular haemorrhage (IVH)

\begin{tabular}{llll}
\hline Measure & $\begin{array}{l}\text { No surgery } \\
(\mathbf{n}=202)\end{array}$ & $\begin{array}{l}\text { Surgery } \\
(\mathrm{n}=19)\end{array}$ & $\mathrm{p}$ Value \\
\hline \% Male & 58.6 & 47.4 & $>0.30$ \\
Gestational age (weeks) & $27.1(2.6)$ & $26.3(1.9)$ & $>0.15$ \\
Birth weight (g) & $1007(293)$ & $940(234)$ & $>0.30$ \\
CRIB score & $5.4(4.2)$ & $7.3(4.3)$ & $>0.05$ \\
$\%$ IVH grade 3 or 4 & 21.2 & 94.7 & $<0.0001$ \\
Max PCO $^{2}$ & $57.2(16.0)$ & $59.9(9.6)$ & $>0.4$ \\
\% Hypertension & 50.3 & 42.1 & $>0.4$ \\
$\%$ Inotropic support & 54.7 & 89.5 & $<0.005$ \\
\% Antenatal corticosteroids & 78.3 & 68.4 & $>0.30$ \\
\hline
\end{tabular}

Where applicable, values are mean (SD). The test of significance was based on the $\chi^{2}$ test of independence for dichotomous measures and one way analysis of variance for continuous measures.

* $\mathrm{PCO}_{2}$ values for nine children who were not ventilated were set to an arbitrary value of 40 . Excluding these nine children does not materially alter the strength of this association.

then including grade of IVH. The first analysis showed that only length of gestation was a significant predictor in the logistic regression model. Further, this variable remained significant when grade of IVH was also entered (table 3 ). The odds ratio for length of gestation suggests that the odds of occurrence of PVD decreased by 0.85 for each week of increase in gestation. Similarly, the odds ratio for grade of IVH suggests that, with each stepwise increase in grade of IVH-for example, from grade 2 to grade 3-the odds of PVD occurring increased more than fivefold.

Comparing the 19 infants who underwent surgical intervention for PVD with the remaining 202 infants with IVH surviving more than 14 days, it is apparent that the only two variables significantly related to the likelihood of surgery were grade of IVH $(p<0.0001)$ and requirement for inotropic sup- port for hypotension $(\mathrm{p}<0.005)$ (table 4). Indeed, all 19 infants requiring surgery had grade 3 or $4 \mathrm{IVH}$, and compared with infants not requiring surgery a significantly greater proportion of these infants had received inotropic support. When both of these variables were entered into a logistic regression model to predict the risk of surgery, only grade of IVH remained significant, suggesting that inotropic support was related to surgical intervention because those receiving such support also tended to have severe IVH.

\section{DISCUSSION}

This cohort of VLBW infants with IVH represents the largest number of infants studied in the late 1990s to examine the incidence of PVD, outline its subsequent course and natural history, and identify predictive factors for adverse short term outcome, defined as requirement for surgical treatment for PVD or death. This sample is characterised by predominantly extremely premature, extremely low birthweight infants surviving in the late 1990s in tertiary care facilities in the United States and New Zealand. The mean (SD) gestational age of 26.8 (2.6) weeks and the mean birth weight of 950 (306) g defines a cohort of smaller, more immature infants than in the cohorts studied in the late 1980s and early 1990s. The more immature infants are at greater risk of severe IVH and subsequent PVD. Indeed, severe grade 3/4 IVH accounted for $80 \%$ of our cases of PVD and was associated with $39 \%$ mortality. It is also noteworthy that the cases of grade 3 IVH were bilateral in $82 \%$ of our cohort, suggesting that these extremely premature infants are at a greater risk of bilateral high grade IVH than previously recognised. ${ }^{59}$ Indeed, 18 of the 19 infants who required surgical treatment exhibited grade 3 or $4 \mathrm{IVH}$, and these 18 infants represented $38 \%$ of all infants surviving for more than 14 days with such severe haemorrhage. Of the 116 infants who survived grade 1 and 2 IVH for more than 14 days, only one ultimately required surgical intervention for PVD.

Concerning the incidence and subsequent course of PVD, we found that $25 \%$ of all infants with IVH developed PVD. This proportion is slightly less than that previously observed. ${ }^{1011}$ However, of the infants who did develop PVD, the course of PVD continued to be aggressive with significant mortality and morbidity. Spontaneous arrest of PVD occurred in only 21 (38\%) of the 56 infants with PVD. Of the $62 \%$ of infants with PVD without spontaneous arrest-that is, persistent PVD$48 \%$ required pharmacological treatment and/or drainage of cerebrospinal fluid by serial lumbar punctures, fully 34\% ultimately required surgery, and $18 \%$ died. Of the 27 infants with persistent PVD treated non-surgically, eight had arrest of their PVD and the remaining 19 required surgical intervention. An additional 21 infants (19\% of PVD) had spontaneous arrest of 
PVD - that is, without intervention. Thus, overall, 52\% of infants with PVD experienced spontaneous or treatment induced arrest compared with $46 \%$ in a previous study, and $34 \%$ ultimately required VP shunt treatment and 18\% died compared with $31-50 \%$ and $19-20 \%$ respectively cited in previous studies. ${ }^{12-14}$ The optimal treatment strategy for posthaemorrhagic ventricular dilatation is not known, but our data reflect differing management plans. Recent studies, published after the period of observation in this study, indicate that neither diuretic treatment nor serial lumbar punctures are beneficial in the management of posthaemorrhagic ventricular dilatation. ${ }^{15-17}$

Concerning predictive factors for adverse short term outcome, including surgical treatment for PVD and death, it is important to note that the indications for surgical treatment of infants with PVD in all the units involved in our study have not changed in recent years. The indications for surgical intervention by placement of either a ventricular reservoir or VP shunt are persistent PVD with signs of increased intracranial pressure and failure to respond to other treatment, including serial lumbar punctures or medical treatment or both. The high incidence of VP shunt insertion in our cohort appears to be related to increased numbers of extremely low birthweight infants ( $<1000 \mathrm{~g}$ ) surviving with severe (grades 3/4) IVH and thereby an increased risk of PVD. Thus, as in previous studies, the risk of adverse outcome, defined in the short term as death or PVD necessitating surgical treatment by VP shunt, was most strongly related to the severity of the initial IVH, with some relation also to earlier gestation. ${ }^{13}$ 18-20

Although other risk factors, including low birth weight, illness severity as quantified by CRIB scores, hypotension necessitating inotropic support, or worse respiratory illness manifested as elevated $\mathrm{PCO}_{2}$, were found to be associated with an increased risk of PVD, unlike previous studies ${ }^{21}$ these variables did not predict the requirement for VP shunt when grade of IVH was taken into account. This suggests that these other risk factors indicate infants at risk of severe IVH. Only grade of IVH $(p<0.0001)$ was significantly related to the likelihood of surgical intervention. Although antenatal administration of corticosteroids has been shown to lead to reduced mortality, respiratory distress syndrome, and IVH in preterm infants, ${ }^{22}{ }^{23}$ in our cohort, there was no significant effect of antenatal steroids on the occurrence of PVD or the need for VP shunt insertion for PVD. This finding may reflect the high background use of antenatal corticosteroids, which were administered to $78 \%$ of mothers in our cohort.

Our study emphasises in this population of extremely premature infants characteristic of the late 1990s that severe IVH is the major predictor of adverse short term outcome, defined as death or the subsequent need for surgical intervention for PVD. Although the overall incidence of IVH in this vulnerable population in modern day neonatal intensive care facilities has not changed appreciably, the severity of the lesion has increased and thereby so has the risk of adverse short term outcome. Conversely, it is rare for an infant with grade 1 or 2 IVH to develop PVD that requires surgical intervention. These data can be useful to the clinician in the anticipatory management of very small premature infants. Future research to find strategies for the prevention of severe IVH will have the greatest impact on prevention of PVD and the requirement for surgical intervention.

\section{Authors' affiliations}

B P Murphy, Division of Newborn Medicine, Brigham and Women's Hospital, Harvard Medical School, Boston, USA

T E Inder, J J Volpe, Department of Neurology, Children's Hospital
Boston, Harvard Medical School, Boston, USA

V Rooks, G A Taylor, Department of Radiology, Children's Hospital Boston

N J Anderson, Department of Radiology, Christchurch Hospital, University of Otago, New Zealand

N Mogridge, Department of Paediatrics, Christchurch Hospital L J Horwood, Christchurch School of Medicine, Christchurch, New Zealand

\section{REFERENCES}

1 Investigators of the Vermont-Oxford Trials Network Database Project. The Vermont-Oxford Trials Network: very low birth weight outcomes for 1990. Pediatrics 1993;91:540-5

2 Vohr BR, Wright LL, Dusick AM, et al. Neurodevelopmental and functional outcomes of extremely low birth weight infants in the National Institute of Child Health and Human Development Neonatal Research Network, 1993-1994. Pediatrics 2000;105:1216-26.

3 Hack $M$, Fanaroff AA. Outcomes of children of extremely low birthweight and gestational age in the 1990's. Early Hum Dev 1999;53:193-218.

4 Randomised trial of early tapping in neonatal posthaemorrhagic ventricular dilatation: results at 30 months. Ventriculomegaly Trial Group. Arch Dis Child Fetal Neonatal Ed 1994;70:129-36.

5 Volpe JJ. Intracranial hemorrhage. In: Volpe JJ, ed. Neurology of the newborn. Philadelphia: WB Saunders Company, 2000:428-93.

6 The CRIB (clinical risk index for babies) score: a tool for assessing initial neonatal risk and comparing performance of neonatal intensive care units. The International Neonatal Network [published erratum appears in Lancet 1993 Sep 4;342:626] [see comments]. Lancet 1993;342:193-8.

7 Davies MW, Swaminathan M, Chuang SL, et al. Reference ranges for the linear dimensions of the intracranial ventricles in preterm neonates. Arch Dis Child Fetal Neonatal Ed 2000;82:F218-23.

8 De Vries LS, Eken P, Dubowitz LM. The spectrum of leukomalacia using cranial ultrasound. Behav Brain Res 1992;49:1-6.

9 Monset-Couchard $M$, de Bethmann O, Brouard-Orzechowski C, et al. Evaluation of peri-intraventricular hemorrhages in newborn infants taking into account laterality and parenchymal location. Application to 323 consecutive cases. J Radiol. 1987;68:159-66.

10 Shankaran S, Slovis TL, Bedard MP, et al. Sonographic classification of intracranial hemorrhage. A prognostic indicator of mortality, morbidity, and short-term neurologic outcome. J Pediatr 1982;100:469-75.

11 Hill A, Volpe JJ. Normal pressure hydrocephalus in the newborn. Pediatrics 1981;68:623-9.

12 Resch B, Gedermann A, Maurer U, et al. Neurodevelopmental outcome of hydrocephalus following intra-/periventricular hemorrhage in preterm infants: short- and long-term results. Childs Nerv Syst 1996;12:27-33.

13 Dykes FD, Dunbar B, Lazarra A, et al. Posthemorrhagic hydrocephalus in high-risk preterm infants: natural history, management, and long-term outcome. J Pediatr 1989;114:611-18.

14 Szymonowicz W, Yu VY, Bajuk B, et al. Neurodevelopmental outcome of periventricular haemorrhage and leukomalacia in infants $1250 \mathrm{~g}$ or less at birth. Early Hum Dev 1986;14:1-7.

15 International randomized controlled trial of acetazolamide and furosemide in posthaemorrhagic ventricular dilatation in infancy. Lancet 1998;352:433-40;

16 Whitelaw A, Kennedy CR, Brion LP. Diuretic therapy for newborn infants with posthemorrhagic ventricular dilatation (Cochrane Review). In: The Cochrane Library. Oxford: Update Software, 2001 ; issue 4.

17 Whitelaw A. Postnatal phenobarbitone for the prevention of intraventricular hemorrhage in preterm infants (Cochrane Review). In: The Cochrane Library. Oxford: Update Software, 2001, issue 4.

18 Ahmann PA, Lazzara A, Dykes FD, et al. Intraventricular hemorrhage in the high-risk preterm infant: incidence and outcome. Ann Neurol 1980;7:118-24.

19 Allan WC, Holt PJ, Sawyer LR, et al. Ventricular dilation after neonatal periventricular-intraventricular hemorrhage. Natural history and therapeutic implications. American Journal of Diseases in Children 1982;136:589-93.

20 Bozynski ME, Nelson MN, Rosati-Skertich C, et al. Two year longitudinal followup of premature infants weighing less than or equal to 1,200 grams at birth: sequelae of intracranial hemorrhage. J Dev Behav Pediatr 1984;5:346-52.

21 Hansen AR, Allred EN, Leviton A. Predictors of ventriculoperitoneal shunt among babies with intraventricular hemorrhage. J Child Neurol 1997; 12:381-6.

22 Effect of corticosteroids for fetal maturation on perinatal outcomes. NIH Consens Statement 1994;12:1-24.

23 Crowley PA. Antenatal corticosteroid therapy: a meta-analysis of the randomized trials, 1972 to 1994 [see comments]. Am J Obstet Gynecol $1995 ; 173: 322-35$ 